\title{
Evolution of radiographic damage in ankylosing spondylitis: a 12 year prospective follow-up of the OASIS study
}

\author{
Sofia Ramiro, ${ }^{1,2}$ Carmen Stolwijk, ${ }^{3,4}$ Astrid van Tubergen, ${ }^{3,4}$ Désirée van der Heijde, ${ }^{5}$ \\ Maxime Dougados, ${ }^{6}$ Filip van den Bosch, ${ }^{7}$ Robert Landewé ${ }^{1,8}$
}

Handling editor Tore K Kvien

- Additional material is published online only. To view please visit the journal online (http://dx.doi.org/10.1136/ annrheumdis-2013-204055).

For numbered affiliations see end of article

\section{Correspondence to} Dr S Ramiro, Department of Clinical Immunology and Rheumatology, Academic Medical Centre, University of Amsterdam, Meibergdreef 9 , PO Box 22660, 1100 DD Amsterdam, The Netherlands; sofiaramiro@gmail.com

Received 1 June 2013 Revised 19 July 2013 Accepted 27 July 2013 Published Online First 16 August 2013

\section{CrossMark}

To cite: Ramiro $S$

Stolwijk $C$, van Tubergen $A$, et al. Ann Rheum Dis

2015;74:52-59.
ABSTRACT

Objectives To describe the evolution of radiographic abnormalities of the spine in patients with ankylosing spondylitis (AS)

Methods Patients with AS were followed prospectively with 2 yearly radiographs for 12 years. The modified Stoke Ankylosing Spondylitis Spine Score (mSASSS) was scored by two readers ( $\mathrm{R} 1$ and $\mathrm{R} 2$ ). New syndesmophytes at uninvolved vertebral corners were computed. Radiographic progression was investigated using generalised estimating equations.

Results 809 radiographs (presenting 520 at 2 yearly intervals) from 186 patients (70\% men, mean age 43 (SD 12) years, mean 20 (SD 12) years since symptom onset and 83\% HLA-B27 positive) were included. Mean mSASSS at baseline was 11.6 (16.2). While the course of progression in individual patients was highly variable, and still occurred in patients with decades of symptom duration, mean 2 year progression was 2.0 (3.5) mSASSS units. Over the entire follow-up, at least one new syndesmophyte was found in 55\% (R1) and 63\% (R2) of patients (38\% (R1) and 39\% (R2) of all intervals). In $24 \%$ of patients (39\% of intervals), there was no progression. A progression $\geq 5$ mSASSS units occurred in $22 \%$ of patients (or in $12 \%$ of intervals). At the group level, a linear time course model fitted the data best, with a constant rate over the entire 12 year interval of 0.98 mSASSS units/year. Radiographic progression occurred significantly faster in men, in HLAB27 positive patients and in patients with a baseline mSASSS $\geq 10$.

Conclusions Long term radiographic progression in AS is highly variable in the individual patient, more severe in HLA-B27 positive men and still occurs after decades of disease. At the group level, however, progression in AS follows an approximately linear course.

\section{INTRODUCTION}

Axial spondyloarthritis (axSpA), which includes both non-radiographic axSpA and ankylosing spondylitis (AS), is a chronic inflammatory rheumatic disease that primarily affects the axial skeletal. Likely as a consequence of inflammation, structural damage, mainly characterised by the development of syndesmophytes, may arise over time, which may lead to ankylosis of the spine. It is generally assumed that the disease process is most active in the early phases of the disease and that it becomes quiescent over time.
Radiographic damage is one of the core outcomes recommended by the Assessment of SpondyloArthritis international Society (ASAS) for follow-up of patients with axSpA. ${ }^{1}$ ASAS recommends routine radiography of the lateral cervical and lumbar spine for assessing damage over time in which a 2 year interval is the shortest period defined for reliable assessment of progression. ${ }^{2}$

Syndesmophyte formation in axSpA is not easily retarded by treatment: biological agents have failed to show retardation in structural damage progres$\operatorname{sion}^{4-6}$ and only non-steroidal anti-inflammatory drugs (NSAIDs) have an inhibitory effect, especially in patients with elevated $\mathrm{C}$ reactive protein (CRP). ${ }^{7-9}$ This emphasises the importance of gaining further insight into radiographic progression, in particular about how it naturally evolves during the course of the disease. Radiographic progression has been assessed in a few short-term studies (2 years ${ }^{10} 11$ or 4 years $^{12}{ }^{13}$ ). One study with a duration of 8 years had a retrospective design and only included hospitalised patients. ${ }^{14}$

The aim of the present prospective study was to further investigate the evolution of radiographic abnormalities in patients with AS during 12 years of follow-up. The course of radiographic progression over time was investigated by longitudinal modelling of time, taking into account factors that may significantly modify the course of progression.

\section{METHODS}

\section{Study population}

The study included patients from the Outcome in Ankylosis Spondylitis International Study (OASIS), a prevalence cohort including 217 consecutive patients with AS from The Netherlands, Belgium and France that began in $1996 .^{15}$ Clinical data were collected every 6 months until year 2, then yearly until year 4 and thereafter biannually. Cervical and lumbar spine radiographs were taken biannually over 12 years, with a total of seven possible time points per patient. For the present study, patients were included if they had at least two subsequent time points with an available radiographic damage score so that radiographic progression could be evaluated. All patients gave informed consent to be included in the study.

\section{Scoring methods}

Radiographs were scored using the modified Stoke Ankylosing Spondylitis Spine Score (mSASSS). ${ }^{16}$ 
According to this method, the anterior vertebral corners (VCs) of the cervical (lower border of C2 to upper border of T1) and lumbar (lower border of T12 to upper border of S1) segments (total of $24 \mathrm{VCs}$ ) are scored in the lateral view only for the presence of erosion and/or sclerosis and/or squaring (1 point), syndesmophyte ( 2 points) and bridging syndesmophyte ( 3 points). Total score per patient ranges from 0 to $72 .{ }^{16}$

Two trained experts (SR and CS) independently scored the radiographs, blinded to the demographic and clinical data but with known chronology as this is the most sensitive method ${ }^{17}$ All of the available films per patient were scored at the same time but by both readers separately.

Only scores of radiographs with $\leq 3$ missing VCs per segment (either cervical or lumbar) were used. Individual missing VCs were imputed (see online supplementary text section 1). Reliability between the two readers (R1 and R2) was explored using Bland and Altman analysis ${ }^{18}$ on the progression intervals of mSASSS. An independent adjudicator (AvT) scored all of the radiographs from patients with at least one score beyond the $95 \%$ level of agreement. Averaged scores per VC of the two primary readers were used and, in case of adjudication, the score of the primary reader closest to the adjudicator.

\section{Radiographic abnormalities}

Status scores (the score at each of the available time points) and progression scores (the difference between the status scores of two time points) were calculated. Two year progression scores refer to the progression occurring within 2 years - that is, status score of one time point minus the status score of the immediately previous time point. Twelve year progression score was computed as the score at year 12 minus the score at baseline. Progression scores were calculated for all patients included in the study and also for those who had an mSASSS at 12 years ('12 year completers') in order to trace eventual bias due to loss of follow-up. The contribution (in per cent) of each of the segments (cervical and lumbar) to the 2 year total mSASSS progression was compared with the expected contribution, assuming a balanced distribution (ie, $50 \%$ in each of the segments). In order to better understand the magnitude of the progression of damage, the available 2 year progression scores were classified in categories of progression: $0 ;>0$ and $<1 ; \geq 1 ; \geq 2 ; \geq 1$ and $<3 ; \geq 3 ; \geq 3$ and $<5$; and $\geq 5$ mSASSS units.

At a reader level, the proportion of 2 year intervals with a given number of new syndesmophytes was calculated, both for all patients and for the ' 12 year completers', and for uninvolved VCs (without a previous syndesmophyte or bridge). The proportion of patients in whom at least one new syndesmophyte was identified during follow-up was also estimated.

For further details on data collection, see online supplementary text section 2 .

\section{Statistical analysis}

Interobserver reliability was assessed by Bland and Altman plots $^{18}$ and by smallest detectable change (SDC) for 2 year progression scores. SDC, based on 95\% limits of agreement, is the smallest change that can be detected in an individual beyond measurement error and was calculated as follows ${ }^{19}$ : SDC $=(1.96 \times$ SDdiff $) /(\sqrt{k} \times \sqrt{2}))$ where SDdiff is the SD of the set of differences in change scores obtained by the readers; $\mathrm{k}$ is the number of readers (here two).

Radiographic progression over time was investigated using generalised estimating equations (GEE). This is a technique for longitudinal analysis which makes use of all available longitudinal data, allows unequal numbers of repeated measurements and has some robustness against deviation from normality. ${ }^{20} 21$ GEE corrects for the within subject correlation and for this it requires an a priori defined 'working' correlation structure. In this study, the 'exchangeable' correlation structure was appropriate because the correlations of the outcome at different time points were approximately equal (Spearman correlation coefficients between 0.80 and 0.97 ). Time was modelled in linear and non-linear (quadratic, cubic, logarithmic, exponential or square root) modes and the best fit was determined using the lowest quasi-likelihood information criterion. Relevant two-, three- and four-way interactions between clinical and demographic characteristics of the patients with time were explored, and if a relevant interaction $(p<0.1)$ was found, progression over time was assessed in the subgroups after stratification. Interactions were tested with HLA-B27 status, gender, symptom duration, baseline mSASSS, NSAID intake (dichotomous variable and ASAS NSAID index ${ }^{22}$ ) and exposure to tumour necrosis $\alpha$ inhibitors (TNFi) during follow-up. An interaction between time and a dichotomous variable reflecting the first and second 6 years of follow-up was also tested in order to assess whether the progression rate changed over time. Because the dependent variable was mSASSS and the independent variable time, the regression coefficient obtained reflects the progression of mSASSS per year. Stata SE V.12 was used (Statacorp, College Station, Texas, USA).

\section{RESULTS}

In total, 186 patients in whom paired x-rays were available were included in the study, with the following baseline characteristics: 70\% men; 83\% HLA-B27 positive; mean age 43 (SD 12) years; mean time since symptom onset 20 (SD 12) years; mean disease duration 11 (SD 9) years; Bath AS Disease Activity Index 3.4 (2.0); and CRP 17 (23) $\mathrm{mg} / \mathrm{L}$ (47\% had an elevated CRP). At baseline, $68 \%$ of patients were exposed to NSAIDs and none to TNFi. Throughout follow-up, $95 \%$ of patients were at some time exposed to NSAIDs and $22 \%$ to TNFi (but before year 8 of follow up, this proportion was 5\%).

Duration of follow-up was, on average, 7.9 (4.0) years per patient (range 2-12 years). A total of 809 radiographs (386 radiographs for the 68 ' 12 year completers') were obtained in which mSASSS could be determined (4.2 (1.7) radiographs per patient (range 2-7)).

Interobserver reliability was 'good' (see online supplementary figure S1). SDC measured across all progression intervals was 2.9.

\section{Progression in mSASSS}

Mean (SD) mSASSS at baseline was 11.6 (16.2) (11.2 (15.7) in the ' 12 year completers'). At baseline, $47 \%$ (R1) and 58\% (R2) of patients had at least one syndesmophyte present: 9\% (R1) and $48 \%$ (R2) had a score of 1 in at least one VC.

Mean mSASSS progressed gradually, from 11.6 (16.1) at baseline $(n=184)$ to a mean value of $24.5(21.7)$ at 12 years $(n=68)$. The mean 2 year progression rate (in 520 intervals) was 2.0 (3.5) (2.2 (3.9) for the '12 year completers'). The mean 2 year progression in the cervical segment was $1.2(2.2)$ and mean progression in the lumbar segment was 0.8 (2.0). Progression was significantly higher in the cervical segment (accounting for 66\% of progression) than in the lumbar segment $(33 \%)(p=0.022)$ (table 1). Progression in the cervical segment was not higher in patients with psoriasis (data not shown). In the ' 12 year completers', mean progression was $11.7(11.5)(\mathrm{n}=64)$.

Over the entire follow-up period, in $24 \%$ of patients and in $18 \%$ of the ' 12 year completers' (and in 39\% of the 2 year intervals) there was no progression in mSASSS. Progression $\geq 1$ 


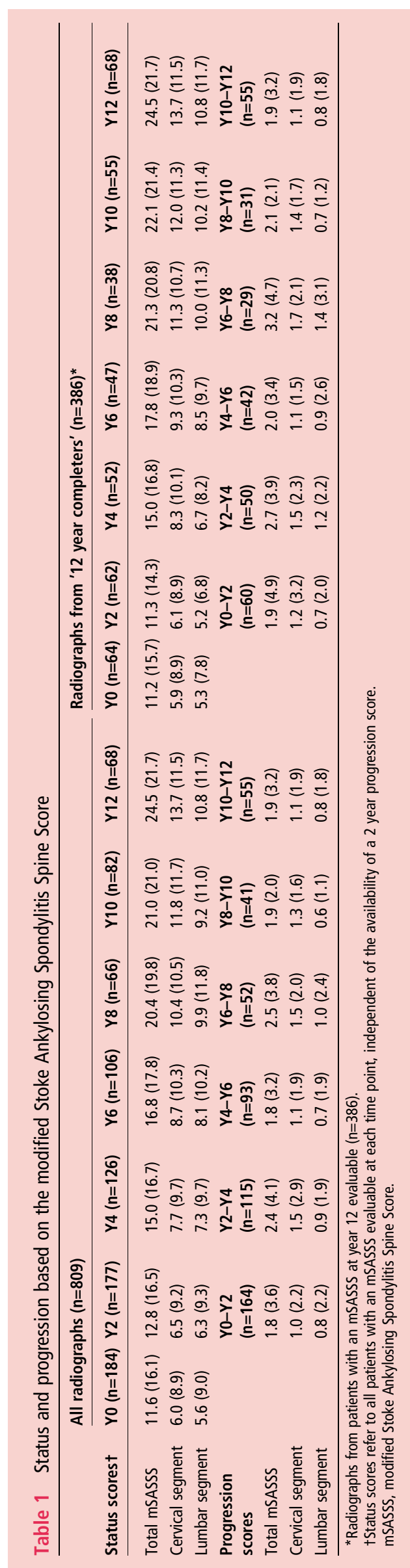

mSASSS unit occurred in $72 \%$ of patients (and in 54\% of the 2 year intervals) and progression $\geq 5$ mSASSS units in $22 \%$ of patients (and in $12 \%$ of the 2 year intervals) (table 2). When taking only the first 2 year interval into consideration, $46 \%$ of patients did not show any mSASSS progression, $48 \%$ showed progression $\geq 2$ units and $25 \%$ showed progression $\geq 5$ units.

\section{Development of new syndesmophytes}

A new syndesmophyte developed in $38 \%$ of all 2 year intervals according to $\mathrm{R} 1$ and in $39 \%$ according to $\mathrm{R} 2$ in previously uninvolved VCs. Throughout follow-up, 55\% (R1) and 63\% (R2) of patients developed at least one syndesmophyte in previously uninvolved VCs (table 3). For the ' 12 year completers', similar results were obtained for the proportion of 2 year intervals with a new syndesmophyte, and in total 69\% (R1) and $74 \%$ (R2) of patients developed at least one syndesmophyte over the 12 years. Taking the first 2 year interval into consideration, 29\% (R1) and 33\% (R2) of patients developed at least one new syndesmophyte.

\section{Radiographic progression over time}

At the individual patient level, radiographic progression was highly variable. In figure $1, \mathrm{mSASSS}$ is plotted as a function of follow-up time (figure 1A) and as a function of symptom duration (figure 1B). Variable progression rates are seen within and across patients, independent of follow-up time and symptom duration (and age-see online supplementary figure S2). This cohort included patients with very short symptom duration as well as patients with more than 40 years of symptom duration. This visual analysis shows that radiographic progression is not only seen in young patients with early disease but also in patients with longstanding disease.

At the group level, however, a linear time function fitted the observed data best (figure 2). Time was positively associated with radiographic damage, at a rate of $0.98 \mathrm{mSASSS}$ units/year. Radiographic progression occurred significantly faster in men than in women (1.11 vs $0.69 \mathrm{mSASSS}$ units/year), in HLA-B27 positive than in HLA-B27 negative patients (1.03 vs 0.70 mSASSS units/year) and in patients with a baseline mSASSS $\geq 10$ (which was the median value) compared with those with a baseline $\mathrm{mSASSS}<10$ (1.44 vs 0.69 mSASSS units/year). HLA-B27 positive men (but not women) had a significantly higher progression than HLA-B27 negative men (1.18 vs 0.69 mSASSS units/year) (interaction of HLA-B27, gender and time: $p=0.17$ ).

HLA-B27 positive men with a baseline mSASSS $\geq 10$ had a higher progression than HLA-B27 negative men with a baseline mSASSS $<10 \quad(1.56$ vs $0.75 \mathrm{mSASSS}$ units/year) (interactions $\mathrm{p}<0.04)$. This effect was not found in women. Progression occurred fully independently of disease and symptom duration (table 4). This table also shows that subgroups of women or HLA-B27 negative patients were small, as usually seen in AS cohorts.

Progression was higher in patients that were ever exposed to TNFi compared with those who were not (1.54 vs $0.82 \mathrm{mSASSS}$ units/year; interaction $\mathrm{p}=0.041$ ).

Progression was independent of treatment with NSAIDs during follow-up, spondyloarthritis related manifestations (psoriasis, inflammatory bowel disease and uveitis) and the presence of a positive spondyloarthritis family history. Progression was not different between the first and the second 6 years of follow-up. 
Table 2 Progression of radiographic damage over time

\begin{tabular}{|c|c|c|c|c|}
\hline Progression* & $\begin{array}{l}\text { No of intervals out of } \\
\text { all } 2 \text { year intervals } \\
(n(\%))(n=520)\end{array}$ & $\begin{array}{l}\text { No of patients during } \\
\text { all } 2 \text { year intervals } \\
\text { (maximum progression)t } \\
(n(\%))(n=186)\end{array}$ & $\begin{array}{l}\text { No of patients from the } \\
\text { '12 year completers' during } \\
\text { all } 2 \text { year intervals } \\
\text { (maximum progression)t } \\
(n(\%))(n=68)\end{array}$ & $\begin{array}{l}\text { No of patients during } \\
\text { the first } 2 \text { year } \\
\text { interval }(Y 0-Y 2) \\
(n(\%))(n=164)\end{array}$ \\
\hline 0 mSASSS units & $204(39)$ & $45(24)$ & $12(18)$ & $75(46)$ \\
\hline$>0$ and $<1$ mSASSS units $\ddagger$ & $0(0)$ & $8(4)$ & $1(1)$ & $11(7)$ \\
\hline$\geq 1$ mSASSS unit & $282(54)$ & $133(72)$ & $54(79)$ & $78(48)$ \\
\hline$\geq 2$ mSASSS units (mean progression)§ & $152(29)$ & $86(46)$ & $39(57)$ & $36(22)$ \\
\hline$\geq 1$ and $<3$ mSASSS units & $161(31)$ & $60(32)$ & $21(31)$ & $20(12)$ \\
\hline$\geq 3$ mSASSS units & $121(23)$ & $73(39)$ & $33(49)$ & $42(26)$ \\
\hline$\geq 3$ and $<5$ mSASSS units & $58(11)$ & $32(17)$ & $12(18)$ & $16(10)$ \\
\hline$\geq 5$ mSASSS units & $63(12)$ & $41(22)$ & $21(31)$ & $41(25)$ \\
\hline
\end{tabular}

\section{DISCUSSION}

The present study, performed in a prevalence cohort of patients with AS, showed that long term radiographic progression in AS is more severe in HLA-B27 positive men, and-as expected-in patients with a higher level of radiographic damage present at baseline. Not less than $60 \%$ of all patients developed at least one new syndesmophyte over a period of up to 12 years.

Radiographic progression follows an approximately linear course at the group level, with a remarkably stable progression rate of approximately $1 \mathrm{mSASSS}$ unit per year when modelled by GEE. More progression was found in the cervical than in the lumbar spine, which is in line with what has been previously reported in an analysis of OASIS with limited follow-up. ${ }^{23}$
The remarkably stable group progression of $1 \mathrm{mSASSS}$ unit per year in AS covers up a highly variable-and to a large extent unpredictable-course of progression of radiographic damage in individual patients. Approximately $25 \%$ of patients showed no progression, $25 \%$ showed a high level of progression (arbitrarily defined as at least one 2 year interval with progression $\geq 5$ mSASSS units) and the remaining patients showed progression rates of about 2 units/ 2 years. But more importantly, simple visualisation of individual progression curves plotted against duration of symptoms revealed that periods of steep progression and relative quiescence may alternate, and this process of oscillation may occur both in 'early AS patients' as well as in 'advanced' patients with ages greatly more than over 60 years.

Table 3 Development of new syndesmophytes over time

\begin{tabular}{|c|c|c|c|c|c|c|}
\hline \multirow[b]{2}{*}{ No of new syndesmophytes } & \multicolumn{2}{|c|}{$\begin{array}{l}\text { All intervals with at least } 1 \\
\text { uninvolved VC* }(n(\%)) \\
(n=468)\end{array}$} & \multicolumn{2}{|c|}{$\begin{array}{l}\text { All intervals with at least } 1 \\
\text { uninvolved VC* in '12 year } \\
\text { completers' }+(\mathrm{n}(\%))(\mathrm{n}=240)\end{array}$} & \multicolumn{2}{|c|}{ 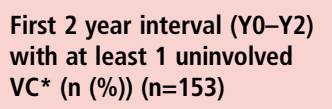 } \\
\hline & Reader 1 & Reader 2 & Reader 1 & Reader 2 & Reader 1 & Reader 2 \\
\hline 0 & $292(62)$ & $286(61)$ & $141(59)$ & $141(59)$ & $109(71)$ & $102(67)$ \\
\hline 1 & $75(16)$ & $95(20)$ & $48(20)$ & $48(20)$ & $16(10)$ & $23(15)$ \\
\hline 2 & $56(12)$ & $52(11)$ & $25(10)$ & $28(12)$ & $19(12)$ & $18(12)$ \\
\hline 3 & $23(5)$ & $15(3)$ & $12(5)$ & $9(4)$ & $4(3)$ & $5(3)$ \\
\hline 4 & $10(2)$ & $9(2)$ & $5(2)$ & $6(3)$ & $2(1)$ & $2(1)$ \\
\hline 5 & $4(1)$ & $4(1)$ & $2(1)$ & $2(1)$ & $2(1)$ & $2(1)$ \\
\hline 6 & $2(0)$ & $2(0)$ & $2(1)$ & $1(0)$ & $0(0)$ & $0(0)$ \\
\hline 7 & $1(0)$ & $2(0)$ & $1(0)$ & $2(1)$ & $0(0)$ & $0(0)$ \\
\hline 8 & $3(1)$ & $2(0)$ & $2(1)$ & $2(1)$ & $0(0)$ & $0(0)$ \\
\hline 12 & $0(0)$ & $1(0)$ & $0(0)$ & $1(0)$ & $0(0)$ & $1(1)$ \\
\hline 13 & $1(0)$ & $0(0)$ & $1(0)$ & $0(0)$ & $1(1)$ & $0(0)$ \\
\hline \multirow[t]{3}{*}{14} & $1(0)$ & $0(0)$ & $1(0)$ & $0(0)$ & $0(0)$ & $0(0)$ \\
\hline & \multicolumn{2}{|c|}{$\begin{array}{l}\text { All patients with at least } \\
1 \text { uninvolved VC (n (\%)) } \\
(n=173)\end{array}$} & \multicolumn{2}{|c|}{$\begin{array}{l}\text { All patients with at least } \\
1 \text { uninvolved VC and with } \\
\text { mSASSS available at Y12 } \\
(n(\%)(n=62)\end{array}$} & \multicolumn{2}{|c|}{ 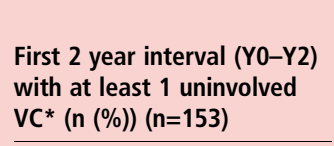 } \\
\hline & Reader 1 & Reader 2 & Reader 1 & Reader 1 & Reader 1 & Reader 2 \\
\hline No of patients with new syndesmophytes & $96(55)$ & $109(63)$ & $43(69)$ & $46(74)$ & $44(29)$ & $51(33)$ \\
\hline
\end{tabular}


Figure 1 Radiographic progression at the patient level. (A) Modified Stoke Ankylosing Spondylitis Spine Score (mSASSS) per patient, plotted as a function of follow-up time. (B) mSASSS plotted as a function of duration since symptom onset.
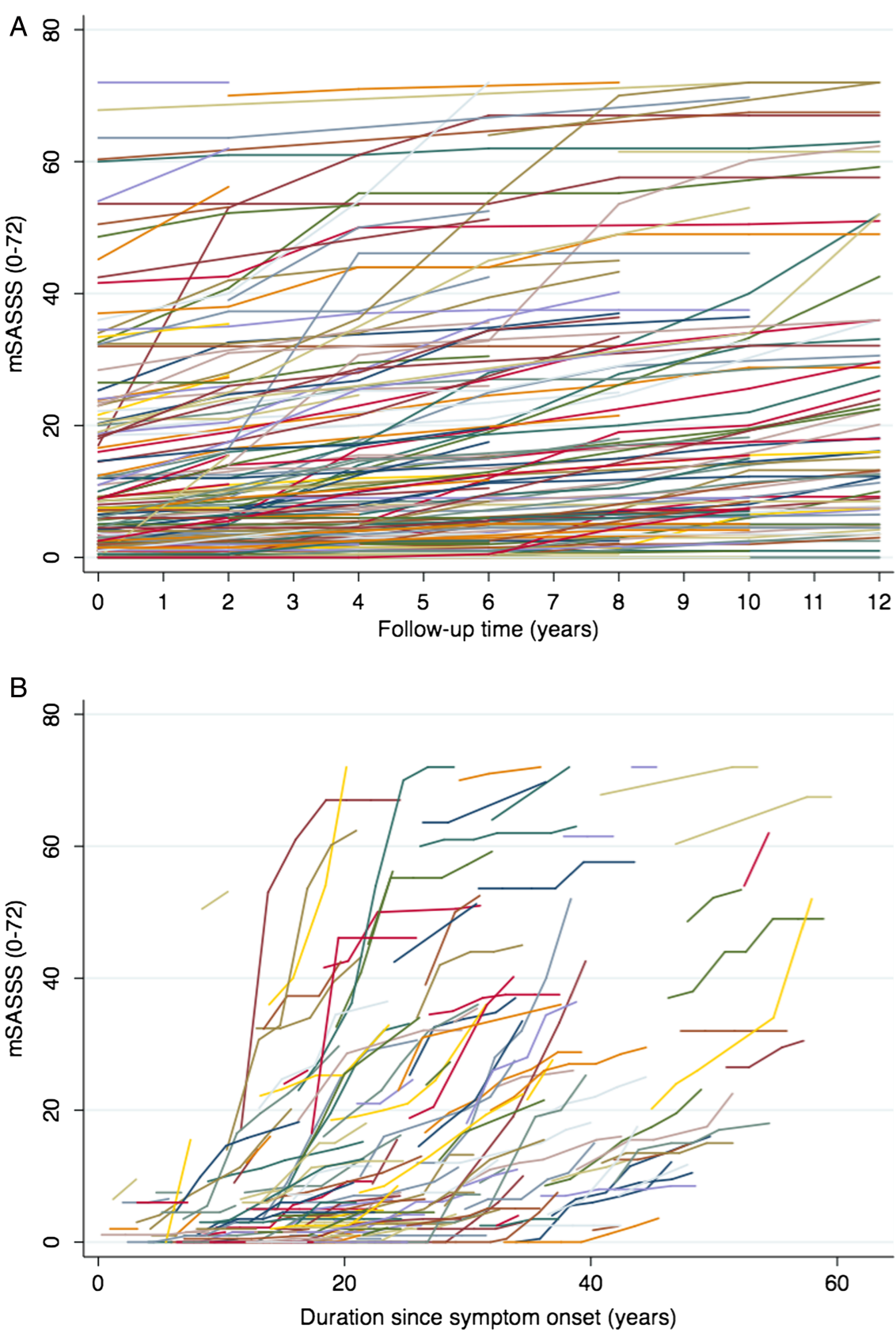

Likely the most important observation of this longitudinal study is that radiographic progression is entirely independent of disease or symptom duration. This observation has implications for clinical practice where a vested opinion says that AS may extinguish over time (which is now demonstrably false), as well as for the necessity of intervening with radiographic progression: 40 years with continuous slow progression or intervals with accelerated progression will sooner or later lead to a situation with clinically important radiographic damage. ${ }^{24}$

We have shown previously that mean progression in OASIS was 1 unit per 2 years while in the current analyses we found a mean progression of 1 unit per year. This at first contradictory result can be fully explained by the difference in reading with blinded time points in the previous analyses and known time points in the current analysis. We have described this effect of (un)blinding time order in a separate paper comparing 2 year progression scores in OASIS. ${ }^{17}$ However, the progression rate reported in this study ( 2 mSASSS units every 2 years) is in line with what has been previously reported in other studies. There are reports of 2 year mSASSS progression scores of approximately 1 mSASSS unit, ${ }^{4-6} 11 \quad 1.5$ units, ${ }^{25} 2.5$ units $^{12}$ and 2.6 units (extrapolation to a 2 year period of the annual progression rate of 1.3$)^{14}$ whereas for the same interval (first 2 year interval) we found a progression rate of 1.8 units. The differences between the scores can be attributed to differences in selection of patients, baseline radiographic damage, reading conditions $^{17}$ or method of imputation of missing VCs. Another study described radiographic damage over time, but only in 20 patients, using the Bath AS Radiology Index, ${ }^{26}$ and thus not allowing a formal comparison. ${ }^{27}$ Furthermore, the mSASSS has been shown to be the most appropriate measure to assess radiographic damage in patients with AS and thus our selection. ${ }^{1328}$

The proportion of patients developing new syndesmophytes over time has not been reported in many studies. Poddubnyy et al reported $11 \%$ of patients with AS with at least one new syndesmophyte and Baraliakos et al reported 19\% of patients, 
Figure 2 Radiographic progression at the group level. mSASSS, modified Stoke Ankylosing Spondylitis Spine Score.

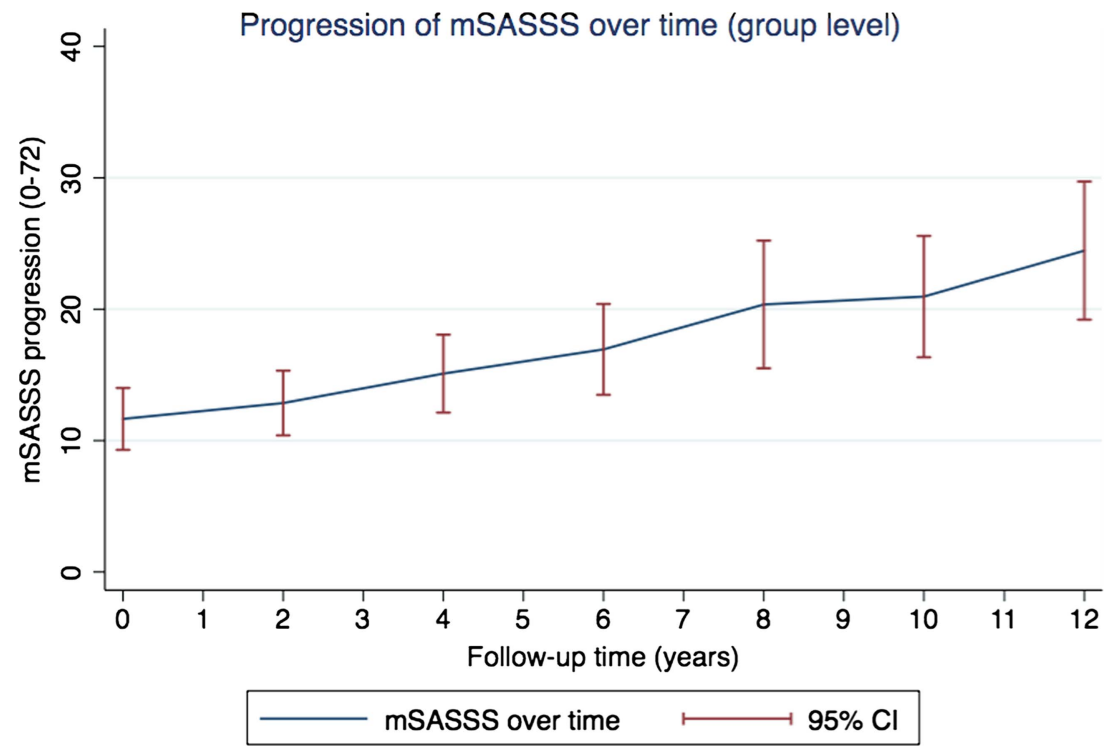

Table 4 Modelling of radiographic progression over time

\begin{tabular}{|c|c|c|c|}
\hline & $\begin{array}{l}\text { Univariable regression } \\
\text { analysis* } \boldsymbol{\beta}(95 \% \mathrm{Cl})\end{array}$ & p Valuet & $\mathrm{N} \neq$ \\
\hline Time (years) & 0.98 (0.84 to 1.12$)$ & & 202 \\
\hline TimexHLAB27 & & 0.052 & 195 \\
\hline Progression rate if HLAB27 negative & $0.70(0.48$ to 0.90$)$ & & 31 \\
\hline Progression rate if HLAB27 positive & $1.03(0.88$ to 1.19$)$ & & 164 \\
\hline Timexgender & & 0.005 & 202 \\
\hline Progression rate in women & $0.69(0.57$ to 0.80$)$ & & 58 \\
\hline Progression rate in men & $1.11(0.93$ to 1.29$)$ & & 144 \\
\hline TimexHLAB27 in women & & 0.958 & 56 \\
\hline TimexHLAB27 in men & & 0.044 & 139 \\
\hline Progression rate in men HLAB27 negative & 0.69 (0.34 to 1.03$)$ & & 19 \\
\hline Progression rate in men HLAB27 positive & 1.18 (0.98 to 1.38$)$ & & 120 \\
\hline Timexbaseline mSASSS & & $<0.001$ & 183 \\
\hline Progression rate in baseline mSASSS $<10$ & $0.69(0.63$ to 0.75$)$ & & 124 \\
\hline Progression rate in baseline mSASSS $\geq 10$ & 1.44 (1.18 to 1.70$)$ & & 59 \\
\hline Timexbaseline mSASSS in women & & $<0.001$ & 55 \\
\hline Progression rate in women with baseline mSASSS $<10$ & $0.49(0.42$ to 0.56$)$ & & 44 \\
\hline Progression rate in women with baseline $\mathrm{mSASSS} \geq 10$ & $1.41(0.97$ to 1.85$)$ & & 11 \\
\hline Timexbaseline mSASSS in men & & $<0.001$ & 128 \\
\hline Progression rate in men with baseline mSASSS $<10$ & $0.81(0.73$ to 0.90$)$ & & 80 \\
\hline Progression rate in men with baseline $\mathrm{mSASSS} \geq 10$ & 1.45 (1.16 to 1.74$)$ & & 48 \\
\hline Timexbaseline mSASSS in HLAB27 negative & & 0.013 & 28 \\
\hline Progression rate in HLA-B27 negative with baseline mSASSS $<10$ & 0.75 (0.63 to 0.86$)$ & & 22 \\
\hline Progression rate in HLA-B27 negative with baseline mSASSS $\geq 10$ & $0.54(-0.20$ to 1.28$)$ & & 6 \\
\hline Timexbaseline mSASSS in HLAB27 positive & & $<0.001$ & 149 \\
\hline Progression rate in HLA-B27 positive with baseline mSASSS $<10$ & $0.68(0.61$ to 0.75$)$ & & 99 \\
\hline Progression rate in HLA-B27 positive with baseline mSASSS $\geq 10$ & 1.54 (1.27 to 1.81$)$ & & 50 \\
\hline Timexbaseline mSASSS in men HLAB27 negative & & 0.011 & 17 \\
\hline Progression rate in HLA-B27 negative men with baseline mSASSS $<10$ & 0.80 (0.63 to 0.98$)$ & & 12 \\
\hline Progression rate in HLA-B27 negative men with baseline mSASSS $\geq 10$ & $0.45(-0.40$ to 1.29$)$ & & 5 \\
\hline Timexbaseline mSASSS in men HLAB27 positive & & $<0.001$ & 107 \\
\hline Progression rate in HLA-B27 positive men with baseline mSASSS $<10$ & $0.82(0.72$ to 0.92$)$ & & 67 \\
\hline Progression rate in HLA-B27 positive men with baseline mSASSS $\geq 10$ & 1.56 (1.25 to 1.87$)$ & & 40 \\
\hline Timexdisease duration & & 0.701 & \\
\hline Timexsymptom duration & & 0.214 & \\
\hline
\end{tabular}


both within a 2 year period. In our study, we found 29-33\% (according to each of the readers) of patients with at least one new syndesmophyte over the same period. These differences can also be explained by the varying presence of baseline syndesmophytes, in all studies the strongest variable to be associated with further progression of damage. ${ }^{11} 1214$

The long term follow-up of our study allows us to conclude that $60 \%$ of all patients develop at least one new syndesmophyte over up to 12 years, implying that syndesmophytes will occur in the vast majority of patients and not only in those with severe radiographic progression.

This study is unique in that it addresses the long term 'natural' course of radiographic progression. The vast majority of OASIS patients has never been exposed to TNFi. Remarkably, in those that have been exposed to TNFi, we found a higher progression rate than in patients that had never been exposed. Likely this is 'confounding by indication': the TNFi exposed patients would be those with more active disease and subsequently higher progression rates. Interestingly, NSAID use, which has been associated with impaired progression, ${ }^{78}$ did not have any impact on radiographic progression over time.

Some limitations of the present study should be addressed. Over the 12 years, almost two-thirds of the patients were lost to follow-up, and selection bias cannot be ruled out. We have presented our results for all patients as well as for the ' 12 year completers' and we have found them to be concordant in every analysis, thus making it unlikely that non-random attrition influenced our observations. OASIS is a prevalence cohort that, despite having a good representation of the spectrum of AS and of patients of varying ages, includes mainly patients with established AS with a relatively high level of baseline damage. Therefore, these results may not be generalisable to patients with short symptom duration. Further, women and HLA-B27 negative patients (especially in combination) do not occur in high numbers in AS cohorts, and estimates of radiographic progression in these subgroups are therefore rather unstable.

Although interobserver reliability of the mSASSS was good, agreement for the individual VC scores between readers at a given time point was worse, mainly because of the long follow-up. However, in most of those cases, readers scored the same new syndesmophytes but at different time points, which is not necessarily the same as non-agreement: if a reader observes the subtle appearance of a new syndesmophyte in a time series of $\mathrm{x}$-rays, he has to decide at which time point that syndesmophyte will first be scored as positive. This methodological phenomenon has precluded an analysis of new syndesmophytes agreed upon by both readers at the same time point. Such an approach would artificially and substantially reduce the number of new syndesmophytes over time.

In summary, over a follow-up period of up to 12 years, progression of structural damage in AS patients is largely unpredictable, often alternating with periods of acceleration and quiescence, and may occur in early as well as in very advanced patients with AS at older ages. About $60 \%$ of all patients developed at least one new syndesmophyte over a period of up to 12 years. Radiographic progression may be underestimated if the data are presented at the group level, as a linear average rate of 1 mSASSS unit per year. In HLA-B27 positive men in particular and in patients with a higher level of damage already present, physicians should be alert to future radiographic progression.

\footnotetext{
Author affiliations

${ }^{1}$ Department of Clinical Immunology and Rheumatology, Academic Medical Centre, University of Amsterdam, Amsterdam, The Netherlands

${ }^{2}$ Department of Rheumatology, Hospital Garcia de Orta, Almada, Portugal
}

${ }^{3}$ Department of Medicine, Division of Rheumatology, Maastricht University Medical Centre, Maastricht, The Netherlands

${ }^{4}$ School for Public Health and Primary Care (CAPHRI), University of Maastricht, Maastricht, The Netherlands

${ }^{5}$ Department of Rheumatology, Leiden University Medical Centre, Leiden, The Netherlands

${ }^{6}$ Rheumatology B Department, Paris-Descartes University, Cochin Hospital, Paris, France

${ }^{7}$ Department of Rheumatology, University of Ghent, Ghent, Belgium

${ }^{8}$ Department of Rheumatology, Atrium Medical Centre, Heerlen, The Netherlands

Contributors SR, AvT, RL and DvdH designed the study. SR, AvT, CS, RL, FvdB, $\mathrm{MD}$ and DvdH collected the data. SR and CS read the radiographs. AvT was the adjudicator. SR, AvT, RL and DvdH analysed the data and critically interpreted the results. SR prepared the first version of the manuscript. All authors reviewed the draft versions and gave their approval of the final version of the manuscript.

\section{Competing interests None}

Funding SR was supported by the Fundação para a Ciência e Tecnologia (FCT) grant SFRH/BD/68684/2010.

Ethics approval The ethics committee from Maastricht University Medical Centre approved the study.

Provenance and peer review Not commissioned; externally peer reviewed.

\section{REFERENCES}

1 van der Heijde $D$, Calin A, Dougados $M$, et al. Selection of instruments in the core set for DC-ART, SMARD, physical therapy, and clinical record keeping in ankylosing spondylitis. Progress report of the ASAS Working Group. Assessments in Ankylosing Spondylitis. J Rheumatol 1999;26:951-4.

2 Zochling J, van der Heijde D, Burgos-Vargas R, et al. ASAS/EULAR recommendations for the management of ankylosing spondylitis. Ann Rheum Dis 2006:65:442-52.

3 Braun J, van den Berg R, Baraliakos X, et al. 2010 update of the ASAS/EULAR recommendations for the management of ankylosing spondylitis. Ann Rheum Dis 2011;70:896-904.

4 van der Heijde $D$, Landewe R, Baraliakos $X$, et al. Radiographic findings following two years of infliximab therapy in patients with ankylosing spondylitis. Arthritis Rheum 2008;58:3063-70.

5 van der Heijde D, Landewe R, Einstein $S$, et al. Radiographic progression of ankylosing spondylitis after up to two years of treatment with etanercept. Arthritis Rheum 2008;58:1324-31.

6 van der Heijde D, Salonen D, Weissman BN, et al. Assessment of radiographic progression in the spines of patients with ankylosing spondylitis treated with adalimumab for up to 2 years. Arthritis Res Ther 2009;11:R127.

7 Wanders A, Heijde D, Landewe R, et al. Nonsteroidal antiinflammatory drugs reduce radiographic progression in patients with ankylosing spondylitis: a randomized clinical trial. Arthritis Rheum 2005;52:1756-65.

8 Poddubnyy $D$, Rudwaleit M, Haibel $H$, et al. Effect of non-steroidal anti-inflammatory drugs on radiographic spinal progression in patients with axial spondyloarthritis: results from the German Spondyloarthritis Inception Cohort. Ann Rheum Dis 2012;71:1616-22.

9 Kroon $F$, Landewe R, Dougados M, et al. Continuous NSAID use reverts the effects of inflammation on radiographic progression in patients with ankylosing spondylitis. Ann Rheum Dis 2012;71:1623-9.

10 Baraliakos $X$, Listing J, Rudwaleit $M$, et al. Progression of radiographic damage in patients with ankylosing spondylitis: defining the central role of syndesmophytes. Ann Rheum Dis 2007;66:910-15.

11 Poddubnyy D, Haibel H, Listing J, et al. Baseline radiographic damage, elevated acute-phase reactant levels, and cigarette smoking status predict spinal radiographic progression in early axial spondylarthritis. Arthritis Rheum 2012;64:1388-98.

12 van Tubergen A, Ramiro S, van der Heijde D, et al. Development of new syndesmophytes and bridges in ankylosing spondylitis and their predictors: a longitudinal study. Ann Rheum Dis 2012;71:518-23.

13 Wanders AJ, Landewe RB, Spoorenberg A, et al. What is the most appropriate radiologic scoring method for ankylosing spondylitis? A comparison of the available methods based on the Outcome Measures in Rheumatology Clinical Trials filter. Arthritis Rheum 2004;50:2622-32.

14 Baraliakos X, Listing J, von der Recke A, et al. The natural course of radiographic progression in ankylosing spondylitis — evidence for major individual variations in a large proportion of patients. J Rheumatol 2009;36:997-1002.

15 Spoorenberg A, van der Heijde D, de Klerk E, et al. Relative value of erythrocyte sedimentation rate and C-reactive protein in assessment of disease activity in ankylosing spondylitis. J Rheumato/ 1999;26:980-4.

16 Creemers MC, Franssen MJ, van't Hof MA, et al. Assessment of outcome in ankylosing spondylitis: an extended radiographic scoring system. Ann Rheum Dis 2005;64:127-9. 
17 Wanders A, Landewe R, Spoorenberg A, et al. Scoring of radiographic progression in randomised clinical trials in ankylosing spondylitis: a preference for paired reading order. Ann Rheum Dis 2004;63:1601-4.

18 Bland JM, Altman DG. Statistical methods for assessing agreement between two methods of clinical measurement. Lancet 1986;1:307-10.

19 Bruynesteyn $K$, Boers $M$, Kostense $P$, et al. Deciding on progression of joint damage in paired films of individual patients: smallest detectable difference or change. Ann Rheum Dis 2005;64:179-82.

20 Twisk J. Applied longitudinal data analysis for epidemiology: a practical guide. Cambridge: Cambridge University Press, 2003.

21 Twisk JW. Longitudinal data analysis. A comparison between generalized estimating equations and random coefficient analysis. Eur J Epidemiol 2004;19:769-76.

22 Dougados M, Simon P, Braun J, et al. ASAS recommendations for collecting, analysing and reporting NSAID intake in clinical trials/epidemiological studies in axial spondyloarthritis. Ann Rheum Dis 2011;70:249-51.

23 van Tubergen $A$, van der Heijde D, Dougados $M$, et al. Are syndesmophytes most prevalent in the lumbar or in the cervical spine in patients with ankylosing spondylitis and do they develop in a specific direction? Rheumatology 2012;51:1432-9.

24 Landewe $\mathrm{R}$, Dougados $\mathrm{M}$, Mielants $\mathrm{H}$, et al. Physical function in ankylosing spondylitis is independently determined by both disease activity and radiographic damage of the spine. Ann Rheum Dis 2009;68:863-7.

25 Baraliakos $X$, Haibel $H$, Listing J, et al. Continuous long-term anti-TNF therapy does not lead to an increase in the rate of new bone formation over 8 years in patients with ankylosing spondylitis. Ann Rheum Dis 2014;73:710-5.

26 MacKay K, Mack C, Brophy S, et al. The Bath Ankylosing Spondylitis Radiology Index (BASRI): a new, validated approach to disease assessment. Arthritis Rheum 1998:41:2263-70.

27 Brophy S, Mackay K, Al-Saidi A, et al. The natural history of ankylosing spondylitis as defined by radiological progression. J Rheumatol 2002;29:1236-43.

28 Ramiro S, van Tubergen A, Stolwijk C, et al. Scoring radiographic progression in ankylosing spondylitis: should we use the modified Stoke Ankylosing Spondylitis Spine Score (mSASSS) or the Radiographic Ankylosing Spondylitis Spinal Score (RASSS)? Arthritis Res Ther 2013;15:R14. 


\section{Correction}

Ramiro S, Stolwijk C, van Tubergen A, et al. Evolution of radiographic damage in ankylosing spondylitis: a 12 year prospective follow-up of the OASIS study. Ann Rheum Dis 2015;74:52-9. In the last column of table 2 the figures are correct, but they have inadvertently been changed between rows. Consequently, the last sentence of the second section of the results (entitled 'Progression in mSASSS') should be adjusted to: 'When taking only the first 2 year interval into consideration, $46 \%$ of patients did not show any mSASSS progression, $25 \%$ showed progression $\geq 2$ units and $12 \%$ showed progression $\geq 5$ units.' The corrected table 2 is given below.

Table 2 Progression of radiographic damage over time

\begin{tabular}{|c|c|c|c|c|}
\hline Progression§ & $\begin{array}{l}\text { Number of intervals } \\
\text { out of all } 2 \text {-year } \\
\text { intervals } \\
(\mathrm{n}(\%))(\mathrm{N}=520)\end{array}$ & $\begin{array}{l}\text { Number of patients during all } \\
\text { 2-year intervals (maximum } \\
\text { progression)* } \\
(\mathrm{n}(\%))(\mathrm{N}=186)\end{array}$ & $\begin{array}{l}\text { Number of patients from the ' } 12 \text {-year } \\
\text { completers' during all } 2 \text {-year intervals } \\
\text { (maximum progression)* } \\
(\mathrm{n}(\%))(\mathrm{N}=68)\end{array}$ & $\begin{array}{l}\text { Number of patients during } \\
\text { the first 2-year interval } \\
(\mathrm{YO}-\mathrm{Y} 2)(\mathrm{n}(\%)) \\
(\mathrm{N}=164)\end{array}$ \\
\hline 0 mSASSS-units & $204(39)$ & $45(24)$ & $12(18)$ & $75(46)$ \\
\hline $\begin{array}{l}>0 \&<1 \\
\text { mSASSS-units** }\end{array}$ & $0(0)$ & $8(4)$ & $1(1)$ & $11(7)$ \\
\hline$\geq 1$ mSASSS-unit & $282(54)$ & $133(72)$ & $54(79)$ & $78(48)$ \\
\hline $\begin{array}{l}\geq 2 \text { mSASSS-units } \\
\text { (mean progression)¥ }\end{array}$ & $152(29)$ & $86(46)$ & $39(57)$ & $41(25)$ \\
\hline$\geq 1 \&<3$ mSASSS-units & $161(31)$ & $60(32)$ & $21(31)$ & $42(26)$ \\
\hline$\geq 3$ mSASSS-units & $121(23)$ & $73(39)$ & $33(49)$ & $36(22)$ \\
\hline$\geq 3 \&<5$ mSASSS-units & $58(11)$ & $32(17)$ & $12(18)$ & $16(10)$ \\
\hline$\geq 5$ mSASSS-units & $63(12)$ & $41(22)$ & $21(31)$ & $20(12)$ \\
\hline
\end{tabular}

mSASSS - modified Stoke Ankylosing Spondylitis Spine Score.

$\S$ Categories of progression are not mutually exclusive.

*Number and proportion of patients, whose maximum 2-year progression score, during follow-up (up to 12 years), met the criterion of each of the rows.

${ }^{* *}$ This category of progression existed because of imputations, which made some status scores and hence progression scores have decimal values.

¥Mean progression taking all 2-year intervals into account.

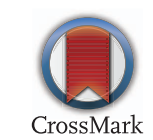

